\title{
ULTRADISCRETIZATION OF THE TZITZEICA EQUATION
}

\author{
RYOGO HIROTA \\ Emeritus Professor, Waseda University, 3-4-1, Ohkubo, Shinjuku-ku, Tokyo 169-8555, Japan \\ e-mail: roy@spn1.speednet.ne.jp \\ and DAISUKE TAKAHASHI \\ Department of Mathematical Sciences, Waseda University, 3-4-1, Ohkubo, Shinjuku-ku, \\ Tokyo 169-8555, Japan \\ e-mail:daisuket@waseda.jp
}

(Received 31 October, 2003; accepted 6 April, 2004)

\begin{abstract}
The trilinear form of the discrete Tzitzeica equation by Schief is found to be a discrete Toda molecule equation with a special boundary condition. Based on this fact, a higher order discrete Tzitzeica equation and an ultradiscrete Tzitzeica equation are obtained.
\end{abstract}

2000 Mathematics Subject Classification. 35Q51.

1. Introduction. Tzitzeica $[\mathbf{1}, \mathbf{2}]$ obtained the equation

$$
(\log h)_{x y}=h-h^{-2}
$$

as the compatibility condition of the Gauss equation

$$
\begin{aligned}
& r_{x x}=\left(h_{x} r_{x}+\lambda r_{y}\right) / h, \\
& r_{x y}=h r, \\
& r_{y y}=\left(h_{y} r_{y}+\lambda^{-1} r_{x}\right) / h,
\end{aligned}
$$

in connection with an affine sphere.

Kaptsov and Shan'ko [3] transformed the Tzitzeica equation, using the dependent variable transformation

$$
v=1-2(\log \tau)_{x y}
$$

into

$$
\left(1-2(\log \tau)_{x y}\right)^{2}\left\{\left(\log \left(\tau^{2}-2 \tau \tau_{x y}+2 \tau_{x} \tau_{y}\right)\right)_{x y}-1\right\}+1=0,
$$

whose numerator is a trilinear equation. They have obtained $\mathrm{N}$-soliton solutions to the trilinear equation.

On the other hand, Schief $[\mathbf{4}, \mathbf{5}]$ has obtained an integrable discrete version of the Tzitzeica equation as the compatibility condition of the discrete Gauss equation,

$$
\begin{aligned}
& \mathbf{r}_{11}-\mathbf{r}_{1}=\alpha\left(\mathbf{r}_{1}-\mathbf{r}\right)+\beta\left(\mathbf{r}_{12}-\mathbf{r}_{1}\right), \\
& \mathbf{r}_{12}+\mathbf{r}=H\left(\mathbf{r}_{1}+\mathbf{r}_{2}\right), \\
& \mathbf{r}_{22}-\mathbf{r}_{2}=\gamma\left(\mathbf{r}_{2}-\mathbf{r}\right)+\delta\left(\mathbf{r}_{12}-\mathbf{r}_{2}\right) .
\end{aligned}
$$


Schief has transformed the discrete Tzitzeica equation into the trilinear form

$$
\begin{aligned}
& \left|\begin{array}{ccc}
\tau(m, n) & \tau(m, n+1) & \tau(m, n+2) \\
\tau(m+1, n) & \tau(m+1, n+1) & \tau(m+1, n+2) \\
\tau(m+2, n) & \tau(m+2, n+1) & \tau(m+2, n+2)
\end{array}\right| \\
& =a b \tau(m+1, n+1)^{3}, \quad a, b \text { being parameters }
\end{aligned}
$$

which is reduced to the following trilinear equation in the continuous limit

$$
\left|\begin{array}{ccc}
\tau & \tau_{x} & \tau_{x x} \\
\tau_{y} & \tau_{x y} & \tau_{x x y} \\
\tau_{y y} & \tau_{x y y} & \tau_{x x y y}
\end{array}\right|=c \tau^{3}, \quad \text { c being a parameter. }
$$

We shall transform the Tzitzeica equation (1) into the bilinear form and find a relation between the trilinear equations (4) and (2). Let $h=G / F$, then we have

$$
\frac{\partial^{2}}{\partial x \partial y} \log h=\frac{G_{x y} G-G_{x} G_{y}}{G^{2}}-\frac{F_{x y} F-F_{x} F_{y}}{F^{2}} .
$$

Here we introduce the bilinear operators $D_{x}$ and $D_{y}$ operating on an ordered pair of $F$ and $G$

$$
\begin{aligned}
D_{x}^{n} F \cdot G & =\left.\left(\frac{\partial}{\partial x}-\frac{\partial}{\partial x^{\prime}}\right)^{n} F(x) G\left(x^{\prime}\right)\right|_{x^{\prime}=x} \\
D_{x}^{n} D_{y}^{m} F \cdot G & =\left.\left(\frac{\partial}{\partial x}-\frac{\partial}{\partial x^{\prime}}\right)^{n}\left(\frac{\partial}{\partial y}-\frac{\partial}{\partial y^{\prime}}\right)^{m} F(x, y) G\left(x^{\prime}, y^{\prime}\right)\right|_{x^{\prime}=x, y^{\prime}=y}
\end{aligned}
$$

which gives

$$
\begin{aligned}
& D_{x} D_{y} F \cdot F=2\left(F_{x y} F-F_{x} F_{y}\right) \\
& D_{x} D_{y} G \cdot G=2\left(G_{x y} G-G_{x} G_{y}\right) .
\end{aligned}
$$

Accordingly the Tzitzeica equation (1) is transformed into

$$
\frac{D_{x} D_{y} G \cdot G-2 \alpha G^{2}+2 F^{2}}{2 G^{2}}-\frac{D_{x} D_{y} F \cdot F-2 \alpha F^{2}+2 G F}{2 F^{2}}=0,
$$

where $\alpha$ is an arbitrary parameter. Accordingly the Tzitzeica equation (1) is decoupled into the bilinear equations

$$
\begin{aligned}
& D_{x} D_{y} G \cdot G=2\left(\alpha G^{2}-F^{2}\right), \\
& D_{x} D_{y} F \cdot F=2\left(\alpha F^{2}-G F\right) .
\end{aligned}
$$

Let us introduce a new dependent variable $\tau_{1}$ and express $F$ and $G$ as follows

$$
\begin{aligned}
& F=\tau_{1}^{2}, \\
& G=\alpha \tau_{1}^{2}-D_{x} D_{y} \tau_{1} \cdot \tau_{1} .
\end{aligned}
$$

Equation (6) is satisfied by this choice of $F$ and $G$, because of the identity

$$
D_{x} D_{y} \tau_{1}^{2} \cdot \tau_{1}^{2}=2 \tau_{1}^{2} D_{x} D_{y} \tau_{1} \cdot \tau_{1}
$$


Let $G=\tau_{2}$, then equations (8) and (5) are written as

$$
\begin{aligned}
& D_{x} D_{y} \tau_{1} \cdot \tau_{1}=\alpha \tau_{1}^{2}-\tau_{2}, \\
& D_{x} D_{y} \tau_{2} \cdot \tau_{2}=2\left(\alpha \tau_{2}^{2}-\tau_{1}^{4}\right) .
\end{aligned}
$$

Equations (9) and (10) are reduced to the trilinear equations (2) and (4) for $\alpha=1$ and $\alpha=0$, respectively, by eliminating $\tau_{2}$.

2. Toda molecule equation. Equations (9) and (10) for $\alpha=0$ remind us of the Toda molecule equation expressed with the bilinear form

$$
D_{x} D_{y} \tau_{n} \cdot \tau_{n}=2 \tau_{n+1} \tau_{n-1},
$$

for $n=1,2, \ldots, N$. For $N=2$ we have

$$
\begin{aligned}
& D_{x} D_{y} \tau_{1} \cdot \tau_{1}=2 \tau_{2} \tau_{0}, \\
& D_{x} D_{y} \tau_{2} \cdot \tau_{2}=2 \tau_{3} \tau_{1},
\end{aligned}
$$

which become equations (9) and (10) for $\alpha=0$ by choosing the boundary condition

$$
\tau_{0}=-1 / 2, \quad \tau_{3}=-\tau_{1}^{3} .
$$

The bilinear equation (11) is transformed into the ordinary form [6]

$$
\frac{\partial^{2}}{\partial x \partial y} \log V_{n}=V_{n+1}-2 V_{n}+V_{n-1},
$$

for $n=1,2, \ldots, N$, through the dependent variable transformation

$$
V_{n}=\frac{\partial^{2}}{\partial x \partial y} \log \tau_{n}=\frac{\tau_{n+1} \tau_{n-1}}{\tau_{n}^{2}} .
$$

Accordingly equations (12) and (13) with the boundary condition (14) are transformed into

$$
\begin{aligned}
& \frac{\partial^{2}}{\partial x \partial y} \log V_{1}=V_{2}-2 V_{1} \\
& \frac{\partial^{2}}{\partial x \partial y} \log V_{2}=4 V_{1}-2 V_{2}
\end{aligned}
$$

Adding twice equation (17) to equation (18) we obtain

$$
\frac{\partial^{2}}{\partial x \partial y} \log \left(V_{1}^{2} V_{2}\right)=0,
$$

which is consistent with

$$
V_{2}=-\frac{1}{4} V_{1}^{-2},
$$

given by equations (14) and (16). 
Substituting equation (20) into equation (17) we obtain

$$
\frac{\partial^{2}}{\partial x \partial y} \log V_{1}=-\frac{1}{4} V_{1}^{-2}-2 V_{1}
$$

Let

$$
V_{1}=-\frac{h}{2}
$$

then equation (21) becomes the Tzitzeica equation

$$
\frac{\partial^{2}}{\partial x \partial y} \log h=h-h^{-2}
$$

Hence we have shown that the Tzitzeica equation is equivalent to the Toda molecule equation with the special boundary condition. The Toda molecule equation is discretized in [7]. Hence the discrete Tzitzeica equation could be obtained by using the discrete Toda molecule equation with a special boundary condition.

The discrete Toda molecule equation [7] is expressed with the bilinear form as follows

$$
\begin{aligned}
& \tau_{s}(m+1, n+1) \tau_{s}(m, n)-\tau_{s}(m+1, n) \tau_{s}(m, n+1) \\
& \quad=q \tau_{s+1}(m, n) \tau_{s-1}(m+1, n+1)
\end{aligned}
$$

for $s=1,2,3, \ldots, N, q$ being a constant, with the boundary conditions

$$
\begin{aligned}
\tau_{0}(m, n) & =1, \\
\tau_{N+1}(m, n) & =f(m) g(n) .
\end{aligned}
$$

We replace the boundary condition (26) by

$$
\tau_{N+1}(m, n)=F(\tau)
$$

in order to obtain the discrete Tzitzeica equation, where $F(\tau)$ is a function of $\tau_{j}$ for $j=1,2, \ldots, N$.

For $N=2$, equation (24) gives

$$
\begin{aligned}
& \tau_{1}(m+1, n+1) \tau_{1}(m, n)-\tau_{1}(m+1, n) \tau_{1}(m, n+1)=q \tau_{2}(m, n), \\
& \tau_{2}(m+1, n+1) \tau_{2}(m, n)-\tau_{2}(m+1, n) \tau_{2}(m, n+1) \\
& \quad=q \tau_{3}(m, n) \tau_{1}(m+1, n+1) .
\end{aligned}
$$

Eliminating $\tau_{2}(m, n)$ from these equations we obtain $\tau_{3}(m, n)$ expressed in terms of $\tau_{1}(m, n)$

$$
\tau_{3}(m, n)=q^{-3}\left|\begin{array}{ccc}
\tau_{1}(m, n) & \tau_{1}(m, n+1) & \tau_{1}(m, n+2) \\
\tau_{1}(m+1, n) & \tau_{1}(m+1, n+1) & \tau_{1}(m+1, n+2) \\
\tau_{1}(m+2, n) & \tau_{1}(m+2, n+1) & \tau_{1}(m+2, n+2)
\end{array}\right| .
$$

Hence equation (30) with the boundary condition

$$
\tau_{3}(m, n)=a b \tau(m+1, n+1)^{3} / q^{3}
$$

is the discrete Tzitzeica equation (4) obtained by Schief. 
3. A Higher order discrete Tzitzeica equation. The discrete Toda molecule equation (24) suggests that we may extend the discrete Tzitzeica equation (4) to a higher order one. We have for $N=3$ and for $\tau_{0}=1$

$$
\begin{aligned}
& \tau_{1}(m+1, n+1) \tau_{1}(m, n)-\tau_{1}(m+1, n) \tau_{1}(m, n+1)=q \tau_{2}(m, n), \\
& \tau_{2}(m+1, n+1) \tau_{2}(m, n)-\tau_{2}(m+1, n) \tau_{2}(m, n+1)=q \tau_{3}(m, n) \tau_{1}(m+1, n+1), \\
& \tau_{3}(m+1, n+1) \tau_{3}(m, n)-\tau_{3}(m+1, n) \tau_{3}(m, n+1)=q \tau_{4}(m, n) \tau_{2}(m+1, n+1) .
\end{aligned}
$$

Eliminating $\tau_{2}$ and $\tau_{3}$ from these equations we obtain

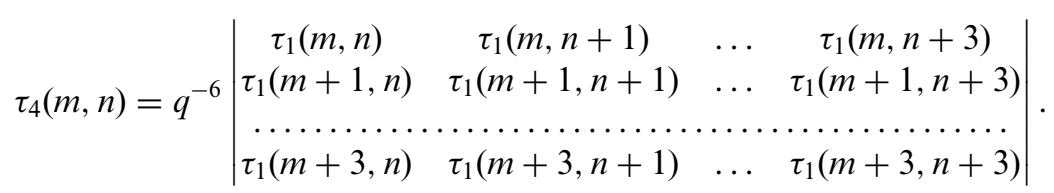

The r.h.s. is a homogeneous polynomial function of $\tau_{1}$ of degree 4 and is invariant under the following gauge transformation:

$$
\tau_{1}(m, n) \rightarrow \tau_{1}(m, n) \exp \left(a_{0} m+b_{0} n\right), \quad a_{0}, b_{0} \text { being constant. }
$$

Taking the gauge invariance into account, we propose the following equation

$$
\begin{gathered}
\left|\begin{array}{cccc}
\tau(m, n) & \tau(m, n+1) & \tau(m, n+2) & \tau(m, n+3) \\
\tau(m+1, n) & \tau(m+1, n+1) & \tau(m+1, n+2) & \tau(m+1, n+3) \\
\tau(m+2, n) & \tau(m+2, n+1) & \tau(m+2, n+2) & \tau(m+2, n+3) \\
\tau(m+3, n) & \tau(m+3, n+1) & \tau(m+3, n+2) & \tau(m+3, n+3)
\end{array}\right| \\
\quad=a b\left|\begin{array}{lll}
\tau(m+1, n+1) & \tau(m+1, n+2) \\
\tau(m+2, n+1) & \tau(m+2, n+2)
\end{array}\right|,
\end{gathered}
$$

as an integrable higher order Tzitzeica equation. The integrability of the equation has not been proved yet. However we have a test of identifying integrable discrete systems proposed by Hietarinta and Viallet $[\mathbf{8}, \mathbf{9}]$ which analyses the complexity (algebraic entropy) of the map using the growth of the degree of its solution. Numerical calculations of the algebraic entropy of the equation indicate the system's integrability.

4. Ultradiscretization of the discrete Tzitzeica equation. In 1990 one of authors (D.T) and Satsuma reported on a simple cellular automaton which shows soliton-like behavior [10]. It is called "soliton cellular automaton". Tokihiro and coworkers have found that the cellular automaton models are obtained as a special limit, known as the ultradiscrete limit, of the integrable equations [11]. Typically, the limiting procedure simply replaces the summation by the Max operator,

$$
\lim _{\varepsilon \rightarrow+0} \varepsilon \log (\exp (A / \varepsilon)+\exp (B / \varepsilon))=\max (A, B)
$$

and the product by the summation,

$$
\lim _{\varepsilon \rightarrow+0} \varepsilon \log (\exp (A / \varepsilon) \times \exp (B / \varepsilon))=A+B .
$$


Let

$$
V_{s}(m, n)=\frac{\tau_{s+1}(m, n) \tau_{s-1}(m+1, n+1)}{\tau_{s}(m+1, n) \tau_{s}(m, n+1)},
$$

then the bilinear form

$$
\begin{aligned}
& \tau_{s}(m+1, n+1) \tau_{s}(m, n)-\tau_{s}(m+1, n) \tau_{s}(m, n+1) \\
& \quad=q \tau_{s+1}(m, n) \tau_{s-1}(m+1, n+1), \quad \text { for } s=1,2, \ldots, N
\end{aligned}
$$

is rewritten as

$$
1+q V_{s}(m, n)=\frac{\tau_{s}(m+1, n+1) \tau_{s}(m, n)}{\tau_{s}(m+1, n) \tau_{s}(m, n+1)}, \quad \text { for } s=1,2, \ldots, N .
$$

Equations (33) and (34) give

$$
\frac{V_{s}(m+1, n+1) V_{s}(m, n)}{V_{s}(m+1, n) V_{s}(m, n+1)}=\frac{\left[1+q V_{s+1}(m, n)\right]\left[1+q V_{s-1}(m+1, n+1)\right]}{\left[1+q V_{s}(m+1, n)\right]\left[1+q V_{s}(m, n+1)\right]},
$$

The boundary condition $\tau_{3}(m, n)=a b \tau_{1}^{3}(m+1, n+1)$ is transformed, using the expression

$$
1+q V_{s}(m, n)=\frac{\tau_{s}(m+1, n+1) \tau_{s}(m, n)}{\tau_{s}(m+1, n) \tau_{s}(m, n+1)},
$$

into

$$
1+q V_{3}(m, n)=\frac{\tau_{3}(m+1, n+1) \tau_{3}(m, n)}{\tau_{3}(m+1, n) \tau_{3}(m, n+1)}=\left[1+q V_{1}(m+1, n+1)\right]^{3} .
$$

Accordingly we have the discrete Tzitzeica equation in the ordinary form,

$$
\begin{aligned}
& \frac{V_{1}(m+1, n+1) V_{1}(m, n)}{V_{1}(m+1, n) V_{1}(m, n+1)}=\frac{\left[1+q V_{2}(m, n)\right]}{\left[1+q V_{1}(m+1, n)\right]\left[1+q V_{1}(m, n+1)\right]}, \\
& \frac{V_{2}(m+1, n+1) V_{2}(m, n)}{V_{2}(m+1, n) V_{2}(m, n+1)}=\frac{\left[1+q V_{1}(m+1, n+1)\right]^{4}}{\left[1+q V_{2}(m+1, n)\right]\left[1+q V_{2}(m, n+1)\right]} .
\end{aligned}
$$

Following the limiting procedure we put

$$
V_{s}(j, k)=\exp \left(x_{s}(j, k) / \epsilon\right), \quad q=\exp (-c / \epsilon), \quad \text { for } s=1,2,3 .
$$

The discrete Tzitzeica is transformed into the following max-plus equations in the small limit of $\epsilon$,

$$
\begin{aligned}
& x_{1}(m+1, n+1)=x_{1}(m+1, n)+x_{1}(m, n+1)-x_{1}(m, n) \\
& \quad+\max \left(0, x_{2}(m, n)-c\right)-\max \left(0, x_{1}(m+1, n)-c\right) \\
& \quad-\max \left(0, x_{1}(m, n+1)-c\right), \\
& x_{2}(m+1, n+1)=x_{2}(m+1, n)+x_{2}(m, n+1)-x_{2}(m, n) \\
& \quad+4 \max \left(0, x_{1}(m+1, n+1)-c\right)-\max \left(0, x_{2}(m+1, n)-c\right) \\
& \quad-\max \left(0, x_{2}(m, n+1)-c\right) .
\end{aligned}
$$


We remark that the discrete sine-Gordon equation is obtained in connection with a discrete geometry [12] as well as the discrete Tzitzeica equation. However it is very difficult to ultradiscretize the former equation in contrast to the latter.

5. Periodic Boundary Conditions. We transform the coordinates $m, n$ into new coordinates $j, k$ :

$$
m=\frac{1}{2}(j+k), \quad n=\frac{1}{2}(j-k)
$$

in order to obtain equations with the periodic boundary condition. We express functions of $m, n$ in the new coordinates

$$
\begin{aligned}
V_{s}(m, n) & =V_{s}(j, k), \quad V_{s}(m+1, n)=V_{s}(j+1, k+1), \\
V_{s}(m, n+1) & =V_{s}(j+1, k-1), \quad V_{s}(m+1, n+1)=V_{s}(j+2, k) .
\end{aligned}
$$

Then, the discrete Tzitzeica equation is transformed into

$$
\begin{aligned}
& \frac{V_{1}(j+2, k) V_{1}(j, k)}{V_{1}(j+1, k+1) V_{1}(j+1, k-1)} \\
& =\frac{\left[1+q V_{2}(j, k)\right]}{\left[1+q V_{1}(j+1, k+1)\right]\left[1+q V_{1}(j+1, k-1)\right]}, \\
& \frac{V_{2}(j+2, k) V_{2}(j, k)}{V_{2}(j+1, k+1) V_{2}(j+1, k-1)} \\
& =\frac{\left[1+q V_{1}(j+2, k)\right]^{4}}{\left[1+q V_{2}(j+1, k+1)\right]\left[1+q V_{2}(j+1, k-1)\right]} .
\end{aligned}
$$

It is generally accepted that the system's integrability is not destroyed by imposing a periodic boundary condition on the system. We use the periodic boundary condition $V_{s}(j, k)=V_{s}(j, k+N)$ in order to transform the partial difference equations (36) and (37) into a coupled form of ordinary difference equations. The algebraic entropy of the higher order discrete Tzitzeica equation (33) is calculated by imposing the periodic boundary condition $V_{s}(j, k)=V_{s}(j, k+N)$ with periods $N=1,3,5, \ldots$.

The discrete Tzitzeica equation is reduced, for $N=1$ (uniform in $k$-direction), to

$$
\begin{aligned}
& \frac{V_{1}(j+2) V_{1}(j)}{V_{1}^{2}(j+1)}=\frac{\left[1+q V_{2}(j)\right]}{\left[1+q V_{1}(j+1)\right]^{2}}, \\
& \frac{V_{2}(j+2) V_{2}(j)}{V_{2}^{2}(j+1)}=\frac{\left[1+q V_{1}(j+2)\right]^{4}}{\left[1+q V_{2}(j+1)\right]^{2}} .
\end{aligned}
$$

Equations (38) and (39) give a relation

$$
\left[\frac{V_{1}(j+3) V_{1}(j+1)}{V_{1}^{2}(j+2)}\right]^{2} \frac{V_{2}(j+2) V_{2}(j)}{V_{2}^{2}(j+1)}=1,
$$

hence

$$
V_{2}(j)=c_{0} c_{1}^{j} V_{1}^{-2}(j+1)
$$


where $c_{0}$ and $c_{1}$ are integration constants. Substituting the relation into equation (38) we obtain

$$
\frac{V_{1}(j+2) V_{1}(j)}{V_{1}^{2}(j+1)}=\frac{\left[1+q c_{0} c_{1}^{j} V_{1}^{-2}(j+1)\right]}{\left[1+q V_{1}(j+1)\right]^{2}} .
$$

Equation (41) is a non-autonomous equation if $c_{1} \neq 1$, while the Tzitzeica equation is an autonomous equation. Hence we choose $c_{1}=1$. Putting $V_{1}(j+1)=x_{n}$ and $c_{0}=q=c$ we obtain a one-dimensional discrete Tzitzeica equation in the following form,

$$
x_{n+1} x_{n-1}=c^{2} \frac{\left(1+x_{n}^{2} / c^{2}\right)}{\left(1+c x_{n}\right)^{2}} .
$$

Let $x_{n}=e^{X_{n} / \epsilon}, c=e^{C / \epsilon}$, then the one-dimesional discrete Tzitzeica equation (42) is transformed into the following max-plus equations in the small limit of $\epsilon$,

$$
X_{n+1}=2 C+2 \max \left(0, X_{n}-C\right)-2 \max \left(0, X_{n}+C\right)-X_{n-1} .
$$

6. Periods of the ultradiscrete Tzitzeica equation. Equation (43) has the following conserved quantity,

$$
H=\max \left(X_{n-1}-X_{n}-2 C,-X_{n-1}+X_{n}-2 C,-X_{n-1}-X_{n}, X_{n-1}+X_{n}\right),
$$

where $H$ is constant for $n$. This quantity $H$ means that equation (43) is integrable. Moreover, a solution from any initial data has the following remarkable features;

(a) If $|(H-2 C) / 4 C|$ is rational, the solution is periodic with a finite period. Otherwise, the period becomes infinite.

(b) The finite period of a solution is determined only by $C$ and $H$.

(c) Therefore, if $C$ is fixed, finite periods of all solutions with the same $H$ are the same.

If we consider equation (43) as a mapping from $\left(X_{n-1}, X_{n}\right)$ to $\left(X_{n}, X_{n+1}\right)$, we can show the above features by a geometrical analysis of solution orbits in a phase plane. We omit the details of the analysis since it needs a long space and only show how a period is determined by $C$ and $H$. Consider the following relation,

$$
\frac{q}{p}=\left|\frac{H-2 C}{4 C}\right|,
$$

where $p$ and $q$ are positive integers and relatively prime. Then the period is expressed by $p$ and $q$ as follows,

$$
\begin{array}{|c|c|c|}
\hline C>0 & H=0 & 2 \\
\cline { 2 - 3 } & 0<H<2 C & 3 p-4 q \\
\cline { 2 - 3 } & H=2 C & 3 \\
\cline { 2 - 3 } & 2 C<H & 3 p+4 q \\
\hline C=0 & 0 \leq H & 4 \\
\hline C<0 & H=2|C| & 1 \\
\cline { 2 - 3 } & 2|C|<H & -3 p+4 q \\
\hline
\end{array}
$$

Note that $0 \leq H$ if $0 \leq C$ and $2|C| \leq H$ if $C<0$, which can be shown from equation (44). 
For example, if $C=3, X_{0}=1$ and $X_{1}=3$, then $H=4$ and $|(H-2 C) / 4 C|=1 / 6$. Consequently we have $q=1$ and $p=6$, and the period becomes $3 p-4 q=14$. Indeed, the solution becomes

$$
X_{n}: 1,3,-7,3,1,-5,5,-1,-3,7,-3,-1,5,-5,1,3, \ldots
$$

If we take the same $C$ and $X_{0}=X_{1}=2$, the same $H, p$ and $q$ are obtained. The solution is

$$
X_{n}: 2,2,-6,4,0,-4,6,-2,-2,6,-4,0,4,-6,2,2, \ldots,
$$

and the period is also 14 .

7. Concluding remarks. We have ultradiscretized the discrete Tzitzeica equation by Schief. The discrete Tzitzeica equation is connected to the discrete affine geometry. So it would be of strong interest to find a geometry connected to the ultradiscrete Tzitzeica equation.

\section{REFERENCES}

1. G. Tzitzeica, C. R. Acad. Sci. 150 (1910), 955-956.

2. G. Tzitzeica, C. R. Acad. Sci. 150 (1910), 1277-1229.

3. O. V. Kaptsov and Yu. V. Shan'ko, Trilinear representation and the Moutard transformation for the Tzitzeica equation, solv-int/9704014 v1 21 Apr. 1997.

4. W. K. Schief, Self-dual Einstein spaces via a permutability theorem for the Tzitzeica equation, Phys. Lett. A 223 (1996), 55-62.

5. W. K. Schief, Self-dual Einstein spaces and a discrete Tzitzeica equation, a permutability theorem link, in Symmetries and Integrability of Difference Equations (Edited by P. A. Clarkson and F. W. Nijhoff) London Mathematical Society Lecture Note Series 225 (1999), 137-148.

6. Ryogo Hirota,The Direct Method in Soliton Theory (in Japanese), (Iwanami,1992).

7. Ryogo Hirota, Discrete Two-Dimensional Toda Molecule Equation, J. Phys. Soc. Japan 56 (1987), 4285-4288.

8. J. Hietarinta and C. -M. Viallet, Singularity confinement and chaos in discrete systems, Phys. Rev. Lett. 81 (1998), 325-328.

9. M. P. Bellon and C. -M. Viallet, Algebraic entropy, chao-dyn/9805006 v3 3 Jun 1998.

10. D. Takahashi and J. Satsuma, A soliton cellular automaton, J. Phys. Soc. Japan 59 (1990), 3514-3519.

11. T. Tokihiro, D. Takahashi, J. Matsukidaira and J. Satsuma, From Soliton Equations to Integrable Cellular Automata through a Limiting Procedure, Phys. Rev. Lett. 76 (1996), $3247-3250$.

12. A. I. Bobenko and U. Pinkall, Discrete surfaces with constant negative Gaussian curvature and the Hirota equation, J. Diff. Geom. 43 (1996), 527-611. 
